\title{
Evaluation of Oxidative Changes in Polycystic Ovary Syndrome Rats with Hypothyroidism Induced by Propylthiouracil
}

\author{
Sara Khodabandeh ${ }^{*}$, Homayoun Khazali, Abdolkarim Hosseini, Vahid Azizi \\ Faculty of Life Sciences and Biotechnology, Shahid Beheshti University, Tehran, Iran
}

\section{*Correspondence to Sara Khodabandeh, Faculty of Life Sciences and Biotechnology, Shahid Beheshti University, Tehran, Iran. Tel: +982129905939, Fax: +982122431664, Email: sa.khodabandeh@mail.sbu.ac.ir}

Received August 15, 2021 Accepted Spetember 20, 2021 Published online September 30, 2021

\begin{abstract}
Introduction: More than 186 million people suffer to infertility worldwide. Polycystic ovary syndrome (PCOS) is the most common cause of chronic anovulation and infertility. PCOS is known as an endocrine disorder in women of marriageable age and is associated with metabolic disorders and dysfunction of the reproductive system. There is also a clear link between fertility and hypothyroidism, which is often associated with ovulation disorders. In recent years, an increasing zest has been witnessed in conducting more research into PCOS and hypothyroidism. So, recognizing the relationship between these two diseases can help for a better understanding of infertility.

Methods: In line with such calls for more research, the present study is aimed at investigating the levels of oxidants and antioxidants in the blood of rats with PCOS induced by estradiol valerate ( $2 \mathrm{mg} / \mathrm{kg}$ of body weight) for 60 days and then received oral propylthiouracil in different doses (1,2 and $4 \mathrm{mg} / \mathrm{kg}$ of body weight) to induce hypothyroidism.

Results: Results showed an increase in catalase (CAT), superoxide dismutase (SOD) and nitric oxide (NO) in PCOS rats. The results also indicated a significant increase in $\mathrm{NO}(P<0.05)$ and a significant decrease in SOD and CAT $(P<0.05)$ after hypothyroidism in rats with PCOS. That is, as the severity of the disease increased, these indicators also showed significant alterations. Conclusion: Hence, hypothyroidism following PCOS exacerbates oxidant and antioxidant imbalances in the body, which can eventually result in tissue damage.

Keywords: Polycystic ovary syndrome, Hypothyroidism, NO, SOD, CAT
\end{abstract}

however. The most obvious association is the increase in body mass index (BMI) and IR witnessed in both diseases. Singla et al contended that the two diseases are linked through inflammatory factors. ${ }^{6}$

Oxidative stress seems to play a pivotal role in the pathogenesis of infertility in both men and women by upsetting the balance of pro-oxidant molecules including reactive oxygen species (ROS) and reactive nitrogen species (RNS) and defense antioxidants. ${ }^{7}$ Disruption of this balance causes instability of cells and reproductive tissues. ${ }^{8}$ There is ample evidence that an increase in inflammatory factors is associated with an increase in the ROS production, and that the two are strongly related to each other and ultimately lead to damage to body tissues by affecting each other. ${ }^{9}$ Unstable radicals are highly reactive

(c) 2021 The Author(s); Published by Zabol University of Medical Sciences. This is an open-access article distributed under the terms of the Creative Commons Attribution License (http://creativecommons.org/licenses/by/4.0), which permits unrestricted use, distribution, and reproduction in any medium, provided the original work is properly cited. 
and supply their required electrons from intracellular biological molecules, namely, lipids, proteins, nucleic acids, and carbohydrates, thereby damaging the cells. ${ }^{10}$

Nowadays, research into the association between PCOS and hypothyroidism has been growing. In 2020, Alahmadi et al, for example, conducted research on rats with PCOS and hypothyroidism and demonstrated the effect of Matricaria chamomilla on improving thyroid function. ${ }^{11}$ Better identifications and understanding of these pathways, it can be argued, not only help impede their occurrences, but also pave the way for treating patients and improving their conditions.

\section{Materials and Methods \\ Animals}

Twenty-five female Wistar rats weighing about $170 \mathrm{~g}$ and having the age of approximately 6 weeks were exposed to a temperature of $22 \pm 2^{\circ} \mathrm{C}$ and a humidity of $50 \pm 5$. The rodents were maintained at alternating periods of 12 hours of light and 12 hours of darkness.

\section{Materials}

For implementing this experiment, estradiol valerate (EV) and propylthiouracil (PTU) (Iranian Hormone Pharmacy) were used. Nitric oxide (NO), superoxide dismutase (SOD), and catalase (CAT) (Navand Salamat Co., Iran) assay kits were used to measure oxidants and antioxidants, respectively.

\section{Methods}

These 25 rats were randomly divided into five groups ( $\mathrm{n}=5$ for each group), namely control group which received nothing, PCOS group which just received EV and PCOS and hypothyroid groups that respectively received 1,2 and $4 \mathrm{mg}$ of PTU (Table 1). First utilized to induce PCOS; that is, $2 \mathrm{mg}$ of EV per $\mathrm{kg}$ of body weight dissolved in olive oil was injected intramuscularly once during the estrus phase. ${ }^{12}$ The animals were then given about 60 days to fully induce PCOS. After this period, the animals received oral PTU proportional to their weight for 7 days. ${ }^{13}$

At the end of the treatment period and after anesthetizing the animals, blood samples were taken and serum samples were prepared; oxidant and antioxidant

Table 1. Groups and Dosage of EV and PTU

\begin{tabular}{lcc}
\hline Groups & EV $^{\mathbf{a}}$ & PTU $^{\mathbf{b}}$ \\
\hline Group 1 & - & - \\
Group 2 & + & - \\
Group 3 & + & $1 \mathrm{mg}$ \\
Group 4 & + & $2 \mathrm{mg}$ \\
Group 5 & + & $4 \mathrm{mg}$ \\
\hline
\end{tabular}

${ }^{a} \mathrm{EV}$ was injected intramuscularly once during the estrus phase (2mg per kg of body weight).

b PTU was given for 7 days based on group's dose. assays were performed according to the kits' protocol.

\section{Statistical analysis}

The results were analyzed using GraphPad Prism 8 software. ANOVA was used for comparing the groups. Next, post-hoc Tukey test was used to identify and compare significantly different means. Data were presented as Mean \pm SEM and all $P$ values were considered significant at $0.05(P<0.05)$.

\section{Results}

\section{CAT Levels in the Different Treatment Groups}

CAT levels vary from group to group. The PCOS group showed an increase compared to the control group, but this increase is not significant. The groups that received PTU showed a decrease compared to the control group, which was significant only in the group that received the lowest amount of PTU $(P<0.05)$; moreover, these reductions were significant $(P<0.05)$ in all groups receiving PTU compared to the PCOS group (Figure 1).

\section{SOD levels in the Different Treatment Groups}

Changes in SOD levels in different groups increased compared to the control group, but this increase was not significant in all groups. The PCOS group showed a significant increase $(P<0.05)$ in SOD compared to the control group. The groups receiving PTU showed a decrease $(P<0.05)$ only in the group receiving the highest amount of PTU compared to the PCOS group (Figure 2).

\section{NO Levels in the Different Treatment Groups}

NO levels increased in all groups compared to the control group, but this increase was not statistically significant in all groups. A significant increase $(P<0.05)$ was observed in the group that received PCOS along with the second

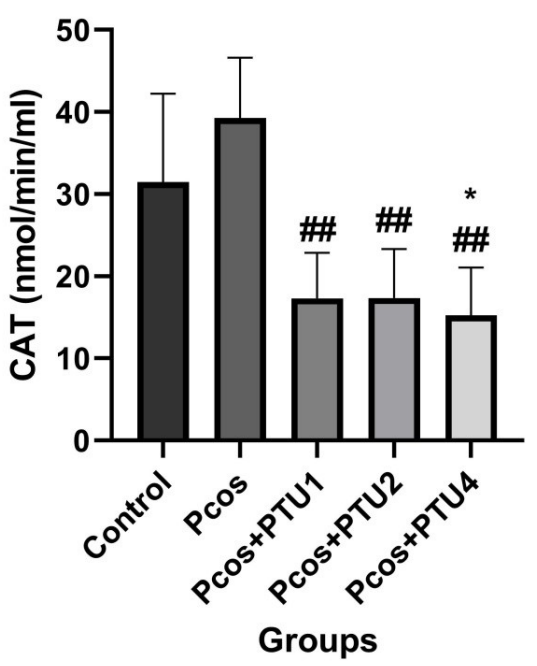

Figure 1. Changes in CAT Levels in Different Groups. Values are signaled as mean \pm SEM. $* P<0.05$ versus control; \#\# $P<0.01$ versus PCOS. Abbreviations: CAT, catalase; PCOS, polycystic ovarian syndrome; PTU, 6-n-propylthiouracil. 


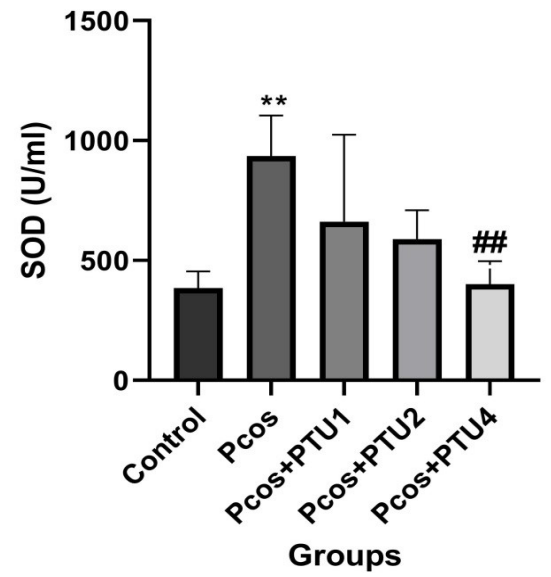

Figure 2. Changes in SOD Levels in Different Groups. Values are mean \pm SEM. ${ }^{* *} P<0.01$ versus control. \#\# $P<0.01$ versus PCOS. Abbreviations: SOD, Superoxide dismutase; PCOS, polycystic ovarian syndrome; PTU, 6-n-propylthiouracil.

dose of PTU compared to the control group. Moreover, the PCOS group that received $4 \mathrm{mg}$ of PTU showed a significant increase $(P<0.05)$ compared to both the control group and the group that was only PCOS (i.e., without receiving anything extra) (Figure 3).

\section{Correlation Between Data}

The correlation between CAT and NO was negative and significant $(P<0.05)$, indicating a perfect reverse relationship between them (Figure 4a). Nevertheless, no significant correlation was observed between SOD and NO (Figure 4b).

\section{Discussion}

What stands out from the result is that CAT, SOD and NO increased in PCOS rats compared with control group, which it was significant only in SOD. On the other hand, CAT and SOD illustrated a gradual decrease in hypothyroid rats in a dose-dependent process. In contrast NO showed a considerable rise in hypothyroid rats. It should be noted that a significant correlation only demonstrated between NO and CAT.

The results of this study demonstrate that the prevalence of hypothyroidism in people with PCOS augments the imbalance between antioxidants and oxidants, increasing oxidative stress in these patients. This imbalance can also eventually lead to damage to the reproductive and thyroid tissues. Therefore, early detection of hypothyroidism in these people can prevent further complications that hamper the exacerbation of the disease and further damage.

As noted, in recent years, several studies have documented an increase in hypothyroidism in women with PCOS. Singla et al., for instance, explained the decrease in thyroid hormone levels that lead to hypothyroidism in people with PCOS. They believed that the increase in TSH secretion in people with PCOS is due to obesity and

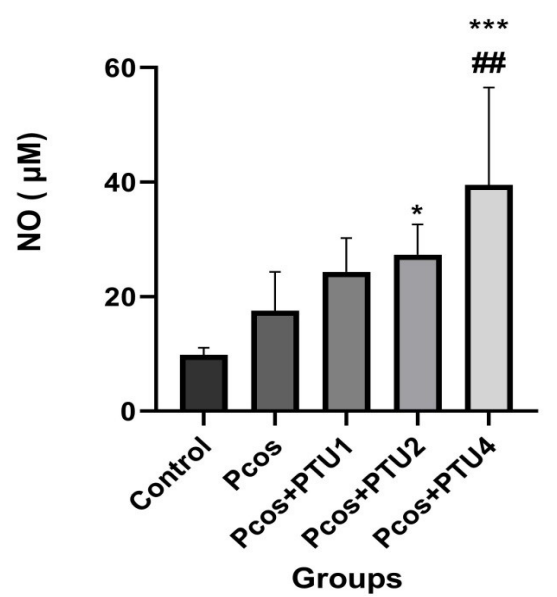

Figure 3. Changes in NO Levels in Different Groups. Values are displayed as mean \pm SEM. $* P<0.05$, *** $P<0.001$ versus control. \#\# $P<0.01$ versus PCOS. Abbreviations: NO, nitric oxide; PCOS, polycystic ovarian syndrome; PTU, 6-n-propylthiouracil.

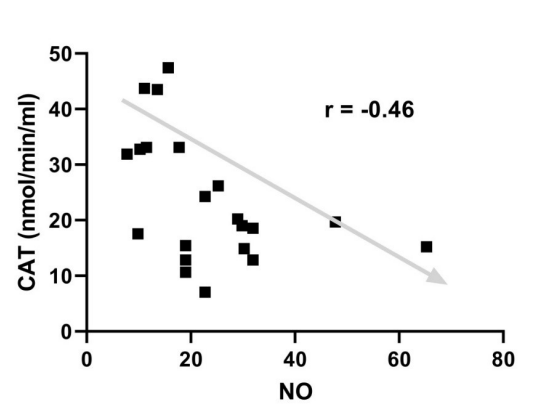

- CAT

b

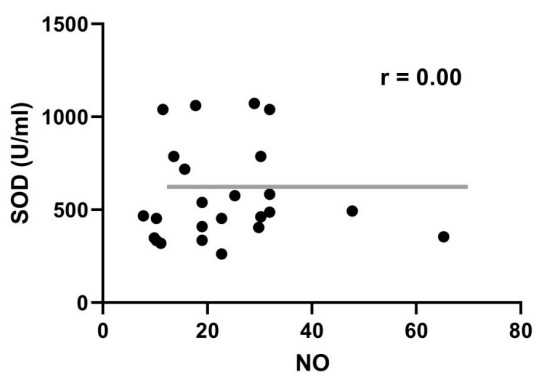

Figure 4. Diagrams of the Correlation Between Oxidants (NO) and Antioxidants (SOD \& CAT). Diagram an exhibit the correlation of NO vs. CAT and diagram b displays NO vs. SOD. Abbreviations: NO, nitric oxide; CAT, catalase; SOD, superoxide dismutase.

supported this by counting two mechanisms. The argued that these mechanisms eventually result in an increase in the pro-inflammatory factors of adipose tissue. Obesity is also associated with increased pro-inflammatory factors. ${ }^{6}$ Increased pro-inflammatory factors are associated with increased ROS-related oxidative stress. ${ }^{14}$

Oxidative stress is caused by upsetting the balance between prooxidants and antioxidants. Overproduction of ROS or RNS or reduction of antioxidant defense mechanisms are two mechanisms that often upset this balance. ${ }^{7}$ PCOS is thought to be due to a decrease in the antioxidants and creation of oxidative state. ${ }^{15}$ On the 
other hand, hypothyroidism is associated with increased ROS and RNS. ${ }^{16}$

The NO molecule is a free radical that acts as a cellular messenger molecule. However, its considerable portion is toxic. ${ }^{17}$ This molecule is produced from the conversion of L-citrulline to L-arginine by enzyme NO synthase. ${ }^{18}$ It is also produced by the immune system through phagocytes such as monocytes, macrophages and neutrophils. ${ }^{17}$

In healthy humans, insulin sensitivity and the rate of endothelial NO synthesis are correlated, and diseases such as PCOS-which cause insulin resistance-affect the NO production. ${ }^{19}$ Erdogan et al, after measuring NO in women with PCOS and in the control group, did not observe any significant difference between the two groups. ${ }^{20}$ Conversely, in 2012, another study was conducted on the relationship between PCOS and oxidative stress. The results of the mentioned study showed that the amount of NO in people with PCOS increased significantly; the results in the mentioned study were consistent with the report provided by Karabulut et al. ${ }^{21}$ In a meta-analysis conducted in 2019, no significant relationship was found between PCOS and NO. The findings of the present study also unveiled an increase in NO in the PCOS group compared to the control group. This increase did not seem to be significant.

In 2001, a study was performed on Behçet's disease or the Silk Road disease and reported that NO levels increased in the immune-inflammatory phase of the disease. $^{22}$ Inhibition of NO synthesis was also found to help improve inflammatory joint disease. ${ }^{23}$ On the other hand, in 2004, it was found that in hypothyroidism, inflammatory cytokines increase. ${ }^{24}$ Baskol et al documented an elevation in the NO levels in patients with hypothyroidism compared to the control group and considered it as a result of increased inflammatory processes. ${ }^{25}$ The results of the present study also showed an increase in NO after induction of hypothyroidism; however, this increase was significant in the groups that received the highest amount of PTU (PTU2, PTU4) visà-vis the control group and in the group that received the highest amount of PTU, namely PTU4, compared to the PCOS group.

SOD is an important enzyme that rapidly converts superoxide $\left(\mathrm{O}_{2}^{-}\right)$to hydrogen peroxide $\left(\mathrm{H}_{2} \mathrm{O}_{2}\right)$; eventually also hydrogen peroxide is converted to water by glutathione peroxidase. Superoxide anion is the result of incomplete reduction of oxygen and the addition of an electron to molecular oxygen. ${ }^{26,27}$

SOD activity in PCOS has been studied in various studies. In 2001, Sabuncu et al, for instance, demonstrated that SOD was higher in patients with PCOS than in that of the control groups, counting compensation for oxidative damage as the cause. ${ }^{28}$ Giving credence to our findings in the present study, Kuşçu \& $\operatorname{Var}^{29}$ and Mohammadi ${ }^{17}$ also documented an increase in SOD. In a meta-analysis conducted in 2013, it was found that $34 \%$ of the average SOD activity in the PCOS group was higher than that of the control group. ${ }^{30}$ However, there are studies that show a decrease in SOD. ${ }^{31,32}$ The results of the present study showed a significant increase in the amount of SOD, and since the amount of NO did not show a significant increase, it is suggested that increasing the amount of SOD inhibits the oxidative activities of NO.

Hypothyroidism is associated with impaired lipid metabolism and causes ramifications by increasing LDL levels. ${ }^{33,34}$ Increased LDL oxidation has been reported in hypothyroidism. ${ }^{35}$ The body produces antioxidant enzymes to protect tissues. ${ }^{36}$ One of them is SOD, which, as mentioned, eliminates $\mathrm{O}_{2}$

Santi et al, by examining the relationship between thyroid hormones and oxidative stress, showed that in people with hypothyroidism, SOD levels increase significantly. ${ }^{37}$ However, there are other studies that have not reported significant changes in the levels of antioxidant enzymes. ${ }^{38,39}$ Baskol et al did not see a significant increase in hypothyroidism compared to the control group, but after treatment with thyroxine (T4), an increase in SOD was observed. In the present study, an increase in SOD is discernable compared to the control group, but this increase is not significant in any of the groups receiving PTU. The results of this study also show a decrease in SOD after receiving PTU; the increase, however, was significant only in the group that received the highest amount of PTU compared to the PCOS group. Alahmadi et al in a similar study showed a reduction in some other antioxidant factors. ${ }^{11}$ According to the results on SOD and NO levels, similar to what was stated by Baskol et al, ${ }^{25}$ SOD is not effective in inactivating $\mathrm{NO}$ after hypothyroidism.

Another antioxidant enzyme is CAT. Various studies have been conducted on its extent in PCOS. However, the results seem to have been incomprehensive or vague. Some studies have reported its increase, ${ }^{40,41}$ some have reported its decrease ${ }^{42}$ and even some have documented its similarity ${ }^{43}$ in the PCOS group compared to the control one, which confuses researchers in interpreting these results. At any rate, the present study also showed a significant increase in CAT.

In a somewhat similar study, Alahmadi and colleagues examined CAT levels in rats with PCOS and hypothyroidism and reported a significant reduction for CAT. ${ }^{11}$ In the present study, a significant decrease in CAT was observed in all groups compared to the PCOS group and in the group that received the highest amount of PTU compared to the control group, which confirms the results of Alahmadi et al. ${ }^{11}$

\section{Conclusion}

Overall, it can be concluded that while hypothyroidism in rats with polycystic ovaries leads to an increase in 
the NO oxidants, it leads to a decrease in the CAT and SOD antioxidants. This imbalance affects the damage to reproductive tissues and even thyroid tissue. Moreover, as it was specified, the increase in the severity of hypothyroidism in the dose-dependent process leads to the deterioration of organs. Thus, measuring these indicators in the early stages of hypothyroidism and PCOS as well as early diagnosing or detecting can impede further damage to the tissues and other organs of the body, especially the reproductive system.

\section{Ethical Approval}

The research design was confirmed from Regional Research Ethics Committees of Shahid Beheshti University, Tehran, Iran (Approval ID: IR.SBU.REC.1400.061).

\section{Competing Interests}

The authors declare that they have no conflict of interest.

\section{Authors' Contribution}

HK and VA contributed to conception and design. SK and AH conducted to all experimental work, data and statistical analysis, and interpretation of data. HK and VA were responsible for overall supervision. SK, HK, AH, and VA drafted the manuscript. All authors read and approved the final manuscript.

\section{Acknowledgment}

The authors thank the collaboration of the Regional Department of Animal Sciences and Marine Biology, Faculty of Life Sciences and Biotechnology, for supporting the project.

\section{References}

1. Hamilton-Fairley D, Taylor A. Anovulation. BMJ. 2003;327(7414):546-549. doi:10.1136/bmj.327.7414.546

2. Boyle JA, Cunningham J, O'Dea K, Dunbar T, Norman RJ. Prevalence of polycystic ovary syndrome in a sample of Indigenous women in Darwin, Australia. Med J Aust. 2012;196(1):62-66. doi:10.5694/mja11.10553

3. Rosenfield RL, Ehrmann DA. The pathogenesis of polycystic ovary syndrome (PCOS): the hypothesis of PCOS as functional ovarian hyperandrogenism revisited. Endocr Rev. 2016;37(5):467-520. doi:10.1210/er.2015-1104

4. Patel S. Polycystic ovary syndrome (PCOS), an inflammatory, systemic, lifestyle endocrinopathy. J Steroid Biochem Mol Biol. 2018;182:27-36. doi:10.1016/j.jsbmb.2018.04.008

5. Sinha U, Sinharay K, Saha S, Longkumer TA, Baul SN, Pal SK. Thyroid disorders in polycystic ovarian syndrome subjects: A tertiary hospital based cross-sectional study from Eastern India. Indian J Endocrinol Metab. 2013;17(2):304-309. doi:10.4103/2230-8210.109714

6. Singla $R$, Gupta $Y$, Khemani $M$, Aggarwal S. Thyroid disorders and polycystic ovary syndrome: an emerging relationship. Indian J Endocrinol Metab. 2015;19(1):25-29. doi:10.4103/2230-8210.146860

7. Agarwal A, Aponte-Mellado A, Premkumar BJ, Shaman A, Gupta S. The effects of oxidative stress on female reproduction: a review. Reprod Biol Endocrinol. 2012;10:49. doi:10.1186/1477-7827-10-49

8. Agarwal A, Said TM, Bedaiwy MA, Banerjee J, Alvarez JG. Oxidative stress in an assisted reproductive techniques setting. Fertil Steril. 2006;86(3):503-512. doi:10.1016/j. fertnstert.2006.02.088

9. Jha JC, Ho F, Dan C, Jandeleit-Dahm K. A causal link between oxidative stress and inflammation in cardiovascular and renal complications of diabetes. Clin Sci (Lond). 2018;132(16):1811-1836. doi:10.1042/cs20171459

10. Agarwal A, Gupta S, Sekhon L, Shah R. Redox considerations in female reproductive function and assisted reproduction: from molecular mechanisms to health implications. Antioxid Redox Signal. 2008;10(8):1375-1403. doi:10.1089/ ars.2007.1964

11. Alahmadi AA, Alzahrani AA, Ali SS, Alahmadi BA, Arab RA, El-Shitany NAE. Both Matricaria chamomilla and metformin extract improved the function and histological structure of thyroid gland in polycystic ovary syndrome rats through antioxidant mechanism. Biomolecules. 2020;10(1):88. doi:10.3390/biom10010088

12. Çınar M, Eryılmaz ÖG. Experimental models of polycystic ovary syndrome. Medeni Med J. 2016;31(1):53-57. doi:10.5222/mmj.2016.053

13. Bhanja S, Chainy GB. PTU-induced hypothyroidism modulates antioxidant defence status in the developing cerebellum. Int J Dev Neurosci. 2010;28(3):251-262. doi:10.1016/j. ijdevneu.2010.01.005

14. González F. Inflammation in polycystic ovary syndrome: underpinning of insulin resistance and ovarian dysfunction. Steroids. 2012;77(4):300-305. doi:10.1016/j. steroids.2011.12.003

15. Palacio JR, Iborra A, Ulcova-Gallova Z, Badia R, Martínez P. The presence of antibodies to oxidative modified proteins in serum from polycystic ovary syndrome patients. Clin Exp Immunol. 2006;144(2):217-222. doi:10.1111/j.13652249.2006.03061.x

16. Mancini A, Di Segni C, Raimondo S, et al. Thyroid hormones, oxidative stress, and inflammation. Mediators Inflamm. 2016;2016:6757154. doi:10.1155/2016/6757154

17. Mohammadi M. Oxidative stress and polycystic ovary syndrome: a brief review. Int J Prev Med. 2019;10:86. doi:10.4103/ijpvm.IJPVM_576_17

18. Robbins RA, Grisham MB. Nitric oxide. Int J Biochem Cell Biol. 1997;29(6):857-860. doi:10.1016/s1357-2725(96)00167-7

19. Kelly CJ, Speirs A, Gould GW, Petrie JR, Lyall H, Connell JM. Altered vascular function in young women with polycystic ovary syndrome. J Clin Endocrinol Metab. 2002;87(2):742746. doi:10.1210/jcem.87.2.8199

20. Erdogan M, Karadeniz M, Berdeli A, Tamsel S, Yilmaz C. The relationship of the interleukin-6-174G > C genepolymorphism with cardiovascular risk factors in Turkish polycystic ovary syndrome patients. Int J Immunogenet. 2009;36(5):283-288. doi:10.1111/j.1744-313X.2009.00867.x

21. Karabulut AB, Cakmak M, Kiran RT, Sahin I. Oxidative stress status, metabolic profile and cardiovascular risk factors in patients with polycystic ovary syndrome. Med Sci. 2012;1(1):27-34.

22. Evereklioglu C, Turkoz Y, Er H, Inaloz HS, Ozbek E, Cekmen M. Increased nitric oxide production in patients with Behçet's disease: is it a new activity marker? J Am Acad Dermatol. 2002;46(1):50-54. doi:10.1067/mjd.2002.118338

23. Stichtenoth DO, Frölich JC. Nitric oxide and inflammatory joint diseases. $\mathrm{Br}$ J Rheumatol. 1998;37(3):246-257. doi:10.1093/rheumatology/37.3.246

24. Weetman AP. Cellular immune responses in autoimmune thyroid disease. Clin Endocrinol (Oxf). 2004;61(4):405-413. doi:10.1111/j.1365-2265.2004.02085.x

25. Baskol G, Atmaca H, Tanriverdi F, Baskol M, Kocer D, Bayram F. Oxidative stress and enzymatic antioxidant status in patients with hypothyroidism before and after treatment. Exp Clin Endocrinol Diabetes. 2007;115(8):522-526. doi:10.1055/s-2007-981457

26. Hashemian Z, Afsharian P. Role of oxidative stress in 
polycystic ovary syndrome. J Shahid Sadoughi Univ Med Sci. 2020;28(5):2635-2647. doi:10.18502/ssu.v28i5.3972

27. Dröge W. Free radicals in the physiological control of cell function. Physiol Rev. 2002;82(1):47-95. doi:10.1152/ physrev.00018.2001

28. Sabuncu T, Vural H, Harma M, Harma M. Oxidative stress in polycystic ovary syndrome and its contribution to the risk of cardiovascular disease. Clin Biochem. 2001;34(5):407-413. doi:10.1016/s0009-9120(01)00245-4

29. Kuş̧̧u NK, Var A. Oxidative stress but not endothelial dysfunction exists in non-obese, young group of patients with polycystic ovary syndrome. Acta Obstet Gynecol Scand. 2009;88(5):612-617. doi:10.1080/00016340902859315

30. Murri $M$, Luque-Ramírez $M$, Insenser $M$, Ojeda-Ojeda $M$, Escobar-Morreale HF. Circulating markers of oxidative stress and polycystic ovary syndrome (PCOS): a systematic review and meta-analysis. Hum Reprod Update. 2013;19(3):268288. doi:10.1093/humupd/dms059

31. Zhang D, Luo WY, Liao H, Wang CF, Sun Y. [The effects of oxidative stress to PCOS]. Sichuan Da Xue Xue Bao Yi Xue Ban. 2008;39(3):421-423. [Chinese].

32. Seleem AK, El Refaeey AA, Shaalan D, Sherbiny Y, Badawy A. Superoxide dismutase in polycystic ovary syndrome patients undergoing intracytoplasmic sperm injection. J Assist Reprod Genet. 2014;31(4):499-504. doi:10.1007/s10815-014-01907

33. Becerra A, Bellido D, Luengo A, Piédrola G, De Luis DA. Lipoprotein(a) and other lipoproteins in hypothyroid patients before and after thyroid replacement therapy. Clin Nutr. 1999;18(5):319-322. doi:10.1016/s0261-5614(98)80031-9

34. Efstathiadou Z, Bitsis S, Milionis HJ, et al. Lipid profile in subclinical hypothyroidism: is L-thyroxine substitution beneficial? Eur J Endocrinol. 2001;145(6):705-710. doi:10.1530/eje.0.1450705

35. Sundaram V, Hanna AN, Koneru L, Newman HA, Falko JM. Both hypothyroidism and hyperthyroidism enhance low density lipoprotein oxidation. J Clin Endocrinol Metab.
1997;82(10):3421-3424. doi:10.1210/jcem.82.10.4315

36. Maggi-Capeyron MF, Cases J, Badia E, et al. A diet high in cholesterol and deficient in vitamin $E$ induces lipid peroxidation but does not enhance antioxidant enzyme expression in rat liver. J Nutr Biochem. 2002;13(5):296-301. doi:10.1016/s0955-2863(01)00222-4

37. Santi A, Duarte MM, Moresco RN, et al. Association between thyroid hormones, lipids and oxidative stress biomarkers in overt hypothyroidism. Clin Chem Lab Med. 2010;48(11):16351639. doi:10.1515/cclm.2010.309

38. Nanda N, Bobby Z, Hamide A, Koner BC, Sridhar MG. Association between oxidative stress and coronary lipid risk factors in hypothyroid women is independent of body mass index. Metabolism. 2007;56(10):1350-1355. doi:10.1016/j. metabol.2007.05.015

39. Erdamar H, Demirci $H$, Yaman $H$, et al. The effect of hypothyroidism, hyperthyroidism, and their treatment on parameters of oxidative stress and antioxidant status. Clin Chem Lab Med. 2008;46(7):1004-1010. doi:10.1515/ cclm.2008.183

40. Dikmen A, Ergenoglu AM, Yeniel AO, Dilsiz OY, Ercan G, Yilmaz $\mathrm{H}$. Evaluation of glycemic and oxidative/antioxidative status in the estradiol valerate-induced PCOS model of rats. Eur J Obstet Gynecol Reprod Biol. 2012;160(1):55-59. doi:10.1016/j.ejogrb.2011.09.042

41. Bausenwein J, Serke H, Eberle K, et al. Elevated levels of oxidized low-density lipoprotein and of catalase activity in follicular fluid of obese women. Mol Hum Reprod. 2010;16(2):117-124. doi:10.1093/molehr/gap078

42. Mohan SK, Priya VV. Lipid peroxidation, glutathione, ascorbic acid, vitamin $\mathrm{E}$, antioxidant enzyme and serum homocysteine status in patients with polycystic ovary syndrome. Biol Med. 2009;1(3):44-49.

43. Kurdoglu Z, Ozkol H, Tuluce Y, Koyuncu I. Oxidative status and its relation with insulin resistance in young non-obese women with polycystic ovary syndrome. J Endocrinol Invest. 2012;35(3):317-321. doi:10.3275/7682 